\title{
Thermodynamic bounds for existence of normal shock in compressible fluid flow in pipes
}

\author{
SERGIO COLLE \\ Laboratory of Energy Conversion Engineering and Energy Technology (LEPTEN), Department of Mechanical Engineering, \\ Federal University of Santa Catarina (UFSC), Trindade, 88040-900 Florianópolis, SC, Brazil
}

Manuscript received on May 17, 2016; accepted for publication on December 19, 2016

\begin{abstract}
The present paper is concerned with the thermodynamic theory of the normal shock in compressible fluid flow in pipes, in the lights of the pioneering works of Lord Rayleigh and G. Fanno. The theory of normal shock in pipes is currently presented in terms of the Rayleigh and Fanno curves, which are shown to cross each other in two points, one corresponding to a subsonic flow and the other corresponding to a supersonic flow. It is proposed in this paper a novel differential identity, which relates the energy flux density, the linear momentum flux density, and the entropy, for constant mass flow density. The identity so obtained is used to establish a theorem, which shows that Rayleigh and Fanno curves become tangent to each other at a single sonic point. At the sonic point the entropy reaches a maximum, either as a function of the pressure and the energy density flux or as a function of the pressure and the linear momentum density flux. A Second Law analysis is also presented, which is fully independent of the Second Law analysis based on the Rankine-Hugoniot adiabatic carried out by Landau and Lifshitz (1959).
\end{abstract}

Key words: Normal shocks in pipes, compressible fluid flow, gas dynamics, Second Law analysis.

\section{INTRODUCTION}

The study of the shock theory in fluid mechanics started with the pioneering work of (Rayleigh, 1899-1920) who discovered that in a steady-state compressible flow of an ideal gas in a straight pipe under the condition of constant linear momentum, defined by $p+\rho V^{2}$, there occurs a normal shock, whenever the fluid speed reaches the sound speed. Rayleigh described the geometric loci of constant linear momentum in the Mollier diagram as a curve that became known as the Rayleigh curve. Fanno (Shapiro 1953), in his doctor thesis, which was submitted to the Real Academy of Engineering of Genova in 1930, proposed a new approach to predict the normal shock in pipes, other than the one due to Rayleigh ${ }^{1}$. In his approach he considered the gas flow under constant energy density flux defined by $h+V^{2} / 2$. In both works, it is assumed that the mass

E-mail: sergio.colle@ufsc.br

${ }^{1}$ His thesis was approved. However, because he was denounced as a jew, his $\mathrm{PhD}$ title was refused. He never recovered the $\mathrm{PhD}$, until his death in 1962. 
flow density defined by $j=\rho V$ is constant. The geometric loci of states of constant values of $h+V^{2} / 2$ as defined by Fanno became known as the Fanno curve. It is well known in gas dynamics that Rayleigh and Fanno curves can cross each other in two distinct points, one of which where the flow is subsonic and the other one where the flow is supersonic. It is hard to find in scientific journals any novelty concerning the theory on the normal shock in pipes, since there is a considerable number of papers and books devoted to the topic of gas dynamics, covering normal and oblique shock in compressible fluid flow, as well as its application to the design of nozzle, turbine blades and supersonic aircraft wings. The book of Anderson (2003) and the e-book of Bar-Meir (2013) report the state of the art of gas dynamics theory and applications to aerodynamics. However, it seems that few works have been published concerning the thermodynamic analysis of the normal shock in pipes. A remarkable analysis is presented in Landau and Lifshitz (1959) where the authors carried out a thermodynamic analysis focusing the normal shock in pipes based on the Hugoniot-Rankine adiabatic. In their work, based on the Second Law of Thermodynamics, they set down thermodynamic inequalities for both, weak and strong normal shocks. They also carried out a thermodynamic analysis of the Fanno flow, from which the Fanno curve is shaped in the entropy-pressure diagram. In spite the fact that the theory beneath the normal shock is well established, the author of the present paper carried out a thermodynamic analysis for the Rayleigh flow, from which, a theoretical duality for the Fanno flow and the Rayleigh flow is shown to exist. It is shown here that there exist a single state in the entropy pressure diagram, at which the Fanno and Rayleigh curves become tangent. The existence of the tangency point enables one to establish a symmetric thermodynamic formalism which provides the basis to obtain a Second Law inequality. This inequality is expressed in terms of the entropy, either as a function of the pressure and the linear momentum density flux $j_{m}=j\left(p+\rho V^{2}\right)$ or as a function of the pressure and the energy density flux $j_{e}=j\left(h+V^{2} / 2\right)$, where $j=\rho V$ is the mass flow density in the pipe, which is assumed to be constant. The entropy inequality enables one to derive two independent inequalities, which are expressed in terms of the fluid specific heats, the isothermal compressibility coefficient, the adiabatic compressibility coefficient, and the thermal expansion coefficient. It is also shown that the entropy discontinuity arising from a weak normal shock implies the entropy discontinuity for a strong normal shock.

\section{FANNO FLOW}

In Landau and Lifshitz (1959), the authors set down thermodynamic propriety relationship for sonic and supersonic states, by assuming that the mass flow density $j=\rho V$ and the energy flux density $h+V^{2} / 2$ are constant along the pipe axis. However, similar conditions can be found by assuming both $j$ and the energy flux density $j_{e}=j\left(h+V^{2} / 2\right)$ constant. Defining $x$ as the axial coordinate of a pipe and $V$ as the average velocity over the cross section of the pipe, it follows,

$$
\left(\frac{\partial j_{e}}{\partial x}\right)_{j_{e}, j}=j \frac{\partial}{\partial x}\left(h+\frac{V^{2}}{2}\right)_{j_{e}, j}=j\left[\left(\frac{\partial h}{\partial x}\right)_{j_{e}, j}+j^{2} v\left(\frac{\partial v}{\partial x}\right)_{j_{e}, j}\right]=0
$$


where $V=j v$ and $v$ is the fluid specific volume. Furthermore, $d h=T d s+v d p$, and therefore $\left(\frac{\partial h}{\partial x}\right)_{j_{e}, j}=T\left(\frac{\partial s}{\partial x}\right)_{j_{e}, j}+v\left(\frac{\partial s}{\partial x}\right)_{j_{e}, j}$. On the other hand, $\left(\frac{\partial v}{\partial x}\right)_{j_{e}, j}=\left(\frac{\partial v}{\partial p}\right)_{s}\left(\frac{\partial p}{\partial x}\right)_{j_{e}, j}+$ $\left(\frac{\partial v}{\partial s}\right)_{p}\left(\frac{\partial s}{\partial x}\right)_{j_{e}, j}$. These identities lead to

$$
\left[T+j^{2} v\left(\frac{\partial v}{\partial s}\right)_{p}\right]\left(\frac{\partial s}{\partial x}\right)_{j_{e}, j}+v\left[1+j^{2}\left(\frac{\partial v}{\partial p}\right)_{s}\right]\left(\frac{\partial p}{\partial x}\right)_{j_{e}, j}=0
$$

It is well known from thermodynamics that, $\left(\frac{\partial v}{\partial s}\right)_{p}=\frac{T \alpha}{c_{p}}$. For gases this derivative is positive, since the thermal expansion coefficient $\alpha=\frac{1}{v}\left(\frac{\partial v}{\partial T}\right)_{p}$ is also positive. Moreover,

$$
j^{2}\left(\frac{\partial v}{\partial p}\right)_{s}=\frac{V^{2}}{v^{2}}\left(\frac{\partial v}{\partial p}\right)_{s}=V^{2} /\left[v^{2}\left(\frac{\partial p}{\partial v}\right)_{s}\right]=-\frac{V^{2}}{c^{2}}
$$

where $c^{2}=\left(\frac{\partial p}{\partial \rho}\right)_{s}=-v^{2}\left(\frac{\partial p}{\partial v}\right)_{s}$ is the sound speed square for the fluid considered. The above identity can be expressed as follows,

$$
1+j^{2}\left(\frac{\partial v}{\partial p}\right)_{s}=1-\frac{V^{2}}{c^{2}}
$$

Eq. (1) gives the derivative of the pressure as follows,

$$
\left(\frac{\partial p}{\partial x}\right)_{j_{e}, j}=-\left[T+j^{2} v\left(\frac{\partial v}{\partial s}\right)_{p}\right]\left(\frac{\partial s}{\partial x}\right)_{j_{e}, j} / v\left[1+j^{2}\left(\frac{\partial v}{\partial p}\right)_{s}\right]
$$

Since the fluid flow is irreversible one has, $\left(\frac{\partial s}{\partial x}\right)_{j_{e}, j}>0$. By considering that the denominator of the above equation does not vanish for $V \neq c$, Eq. (2) and Eq. (3) imply that $\left(\frac{\partial p}{\partial x}\right)_{j_{e}, j}<0$ for $V<c$ while for $V>c,\left(\frac{\partial p}{\partial x}\right)_{j_{e}, j}>0$. The conclusion is that for a subsonic flow the pressure decreases downstream while for a supersonic flow the pressure increases downstream. Eq. (1) enables one to write the following identity,

$$
\left(\frac{\partial s}{\partial p}\right)_{j_{e}, j}=-v\left[1+j^{2}\left(\frac{\partial v}{\partial p}\right)_{s}\right] /\left[T+j^{2} v\left(\frac{\partial v}{\partial s}\right)_{p}\right]
$$

From Eq. (2) the above identity can be expressed as follows,

$$
\left(\frac{\partial s}{\partial p}\right)_{j_{e}, j}=-v\left(1-\frac{V^{2}}{c^{2}}\right) /\left[T+j^{2} v\left(\frac{\partial v}{\partial s}\right)_{p}\right]
$$

Since the denominator of this equation is positive, one can conclude that for subsonic flow the entropy derivative is negative, while for supersonic flow it is positive. For $V=c$ the above derivative clearly 
vanishes, which shows that the entropy reaches an extremum. These results allow one to conclude that the geometric loci of states of constant $j_{e}$ in the $s-p$ diagram display a curve along which the entropy increases up to the point where the flow is sonic, and decreases thereafter. By using analogous procedure of Landau and Lifshitz, the following identity can be obtained,

$$
\left.\left(\frac{\partial^{2} s}{\partial p^{2}}\right)_{j_{e}, j}\right|_{V=c}=-j^{2} v\left(\frac{\partial^{2} v}{\partial p^{2}}\right)_{s} /\left[T+j^{2} v\left(\frac{\partial v}{\partial s}\right)_{p}\right]
$$

The second derivative of the volume in the above equation can be reduced with the help of a Maxwell relation derived from the Gibbs thermodynamic potential, to obtain the following identity,

$\left(\frac{\partial^{2} v}{\partial p^{2}}\right)_{s}=\frac{\partial}{\partial p}\left(\frac{\partial v}{\partial p}\right)_{s}=\frac{\partial}{\partial p}\left(-k_{s} v\right)_{s}$, where $k_{s}$ is the adiabatic compressibility coefficient. Thus the following identity holds,

$$
\left(\frac{\partial^{2} v}{\partial p^{2}}\right)_{s}=v\left[k_{s}^{2}-\left(\frac{\partial k_{s}}{\partial T}\right)_{p} \frac{\alpha v}{c_{p} T}-\left(\frac{\partial k_{s}}{\partial p}\right)_{T}\right]
$$

where $\alpha$ is the thermal expansion coefficient. For a perfect gas $k_{s}=1 /(k p)$ where $k=c_{p o} / c_{v o}$ is the Poisson coefficient, which is known to be constant. Therefore the derivative of $k_{s}$ respective to the temperature vanishes. Thus Eq. (7) is reduced to the following expression,

$$
\left(\frac{\partial^{2} v}{\partial p^{2}}\right)_{s}=v\left(k_{s}^{2}+\frac{1}{k p^{2}}\right)>0
$$

As remarked in (Landau and Lifshitz 1959), for most real gases of practical interest this derivative is known to be positive. Thus the second derivative given by Eq. (6) is negative at the point for which $V=c$. Therefore, the sonic state is the geometric locus of a maximum for the entropy as a function of $p$, under the condition of constant energy flux density. The above results allow one to justify the Fanno curve shape in the $s-p$ diagram as shown in Fig. 1 reported in the above mentioned reference. The identities given by Eq. (1) to Eq. (6) are shown here to be equivalent to the respective identities reported in the above mentioned reference.

\section{RAYLEIGH FLOW}

The theory presented in the previous section, can be extended to the Rayleigh flow by assuming both, $j$ and the linear momentum density $j_{m}=j\left(p+\rho V^{2}\right)$ constant. By replacing $\rho V^{2}=j^{2} v$ into the last equation it follows, $d\left(p+\rho V^{2}\right)=d\left(p+j^{2} v\right)=d j_{m}$. Thus the following identity holds,

$$
d p+j^{2} d v=0
$$

The above identity leads to the identity, $\left(\frac{\partial p}{\partial x}\right)_{j_{m}, j}+j^{2}\left(\frac{\partial v}{\partial x}\right)_{j_{m}, j}=0$. By replacing the volume derivative by the identity given by, $\left(\frac{\partial v}{\partial x}\right)_{j_{m}, j}=\left(\frac{\partial v}{\partial s}\right)_{p}\left(\frac{\partial s}{\partial x}\right)_{j_{m}, j}+\left(\frac{\partial v}{\partial p}\right)_{s}\left(\frac{\partial p}{\partial x}\right)_{j_{m}, j}$ the following identity is found,

$$
\left(\frac{\partial p}{\partial x}\right)_{j_{m}, j}=-j^{2}\left(\frac{\partial v}{\partial s}\right)_{p}\left(\frac{\partial s}{\partial x}\right)_{j_{m}, j} /\left[1+j^{2}\left(\frac{\partial v}{\partial p}\right)_{s}\right]
$$




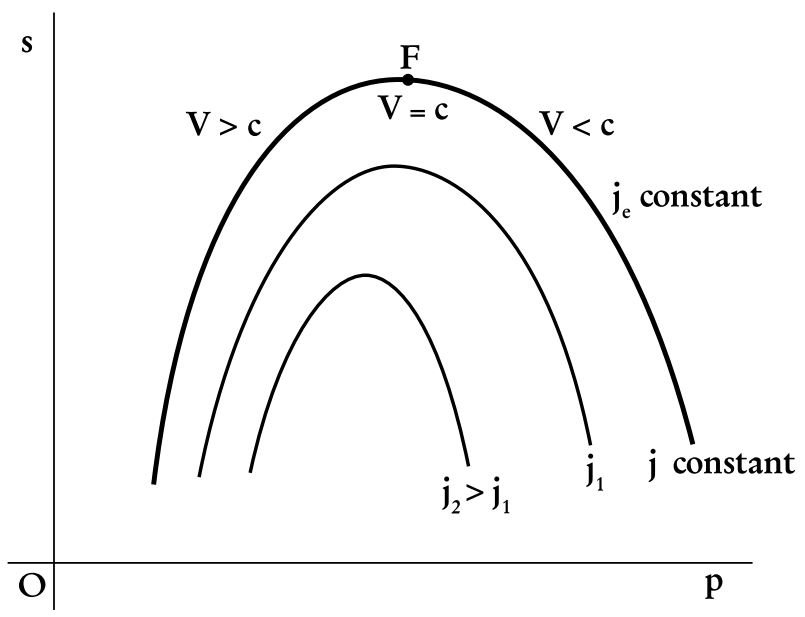

Figure 1 - Fanno curves in the entropy - pressure diagram.

From the above equation and Eq. (2), one can see that $\left(\frac{\partial p}{\partial x}\right)_{j_{m}, j}$ is negative whenever $V<c$ and positive whenever $V>c$. Therefore, the pressure decreases downstream whenever the fluid follow is subsonic and it increases downstream whenever the fluid flow is supersonic. Equation (10) enables one to write the following,

$$
\left(\frac{\partial s}{\partial p}\right)_{j_{m}, j}=-\left[1+j^{2}\left(\frac{\partial v}{\partial p}\right)_{s}\right] /\left[j^{2}\left(\frac{\partial v}{\partial s}\right)_{p}\right]
$$

As has been remarked earlier, the derivative $\left(\frac{\partial v}{\partial s}\right)_{p}$ is positive. It follows from the above equation and Eq. (2) that $\left(\frac{\partial s}{\partial p}\right)_{j_{m}, j}$ is positive whenever $V>c$ and negative whenever $V<c$.

For $V=c$ Eq. (2) implies that $\left(\frac{\partial s}{\partial p}\right)_{j_{m}, j}=0$. Thus, the entropy as a function of $p$ and $j_{m}$ reaches an extremum at the state of sonic flow on the curve of $j_{m}$ constant. Analogous proof as given by Landau and Lifshitz is reported in Colle (2015), to obtain the following identity,

$$
\left.\left(\frac{\partial^{2} s}{\partial p^{2}}\right)_{j_{m}, j}\right|_{V=c}=-\left(\frac{\partial^{2} v}{\partial p^{2}}\right)_{s} /\left(\frac{\partial v}{\partial s}\right)_{p}
$$

As remarked earlier, the derivative $\left(\frac{\partial^{2} v}{\partial p^{2}}\right)_{s}$ is positive. From the above identity it follows that $\left(\frac{\partial^{2} s}{\partial p^{2}}\right)_{j_{m}, j}<0$. Thus, the entropy reaches its maximum as a function of $p$ under the condition of constant linear momentum density flux. The above results indicate that the Rayleigh curve shape is similar to the Fanno curve shape in the $s-p$ diagram of Fig. 1 . 


\section{MAXIMUM ENTROPY THEOREM}

In order to show the existence of a thermodynamic symmetry between Rayleigh flow and Fanno flow, in terms of the entropy either as a function of the pressure and $j_{e}$ or as a function of the pressure and $j_{m}$, under the condition of constant mass flow density, the following Lemma is proposed and proved.

Lemma: If a compressible steady-state axial flow of a gas in a pipe of constant cross section is adiabatic, then the entropy variation along the pipe axis is due to the variation of the linear momentum density flux along the pipe axis. Conversely, if the linear momentum density flux is constant along the pipe axis, then the entropy variation along the pipe axis is due to the heat flux at the boundary of the pipe cross section.

Proof: By assuming $j$ constant, we can write,

$$
\left.d j_{e}\right|_{j}-\left.v d j_{m}\right|_{j}=\left.j d\left(h+V^{2} / 2\right)\right|_{j}-\left.j v d\left(p+\rho V^{2}\right)\right|_{j}
$$

By the definitions of $j_{e}$ and $j_{m}$, the following differential expressions are obtained,

$$
\left.d j_{e}\right|_{j}=\left.d\left(h+j^{2} v^{2} / 2\right)\right|_{j}=d h+j^{2} v d v, \quad \text { and }\left.\quad d j_{m}\right|_{j}=\left.d\left(p+j^{2} v\right)\right|_{j}=d p+j^{2} d v
$$

From these identities it follows,

$$
\left.d j_{e}\right|_{j}-\left.v d d j_{m}\right|_{j}=j\left(d h+j^{2} v d v\right)-j v\left(d p+j^{2} d v\right)=j(d h-v d p)=j T d s
$$

Thus the following thermodynamic identity holds,

$$
\left.d j_{e}\right|_{j}-\left.v d j_{m}\right|_{j}=j T d s
$$

From this identity, the following identities hold

$$
\left(\frac{\partial j_{e}}{\partial x}\right)_{j}-v\left(\frac{\partial j_{m}}{\partial x}\right)_{j}=j T\left(\frac{\partial s}{\partial x}\right)_{j}
$$

The above equation implies the following identities,

$$
\begin{aligned}
& \left(\frac{\partial j_{m}}{\partial x}\right)_{j_{e}, j}=-j T\left(\frac{\partial s}{\partial x}\right)_{j_{e}, j} / v \\
& \left(\frac{\partial j_{e}}{\partial x}\right)_{j_{m}, j}=j T\left(\frac{\partial s}{\partial x}\right)_{j_{m}, j}
\end{aligned}
$$

The first part of the lemma follows from Eq. (15). The second part of the lemma follows from Eq. (16), since $j_{m}$ is constant while $\left(\frac{\partial s}{\partial x}\right)_{j_{m}, j}$ is due to the axial variation of $j_{e}$, which by its turn is proportional to the heat flux variation at the pipe cross section boundary. In other words, quasi-static heat income is required in order to maintain $j_{m}$ constant along the pipe axis downstream. However, it remains to be shown that $j_{m}$ decreases downstream. By the way, for the present case the First Law of thermodynamics, can be expressed by the following equation,

$$
\frac{d}{d x}\left[\rho V\left(h+\frac{V^{2}}{2}\right)\right]_{j}=\left(\frac{\partial j_{e}}{\partial x}\right)_{j}=-\frac{1}{A} \oint_{\Gamma} \hat{q} \cdot \hat{n}_{l} d l
$$


The Second Law can be expressed as follows,

$$
j T\left(\frac{\partial s}{\partial x}\right)_{j}=-\frac{1}{A} \oint_{\Gamma} \hat{q} \cdot \hat{n}_{l} d l+\frac{1}{A} \oint_{A}\left(\dot{\phi}-\frac{\hat{q} \cdot \nabla T}{T}\right) d A
$$

where $A$ is the pipe cross section area, $\hat{q}$ is the heat flux vector, $\hat{n}_{l}$ is the unit vector normal to the cross section boundary curve $\Gamma$, and $\dot{\phi}$ is the fluid power dissipation per unit volume due to friction. From Eq. (14), Eq. (17), and Eq. (18) the following identity is obtained,

$$
-v\left(\frac{\partial j_{m}}{\partial x}\right)_{j}=\frac{1}{A} \int_{A}\left(\dot{\phi}-\frac{\hat{q} \cdot \nabla T}{T}\right) d A
$$

Since from thermodynamics $\hat{q} \cdot \nabla T$ is known to be non-positive, the above equation implies that the linear momentum density decreases with $x$ downstream.

Since Eq. (19) holds regardless to the fact that the flow is adiabatic or not, for an adiabatic flow one has,

$$
-v\left(\frac{\partial j_{m}}{\partial x}\right)_{j}=\frac{1}{A} \int_{A}\left(\dot{\phi}-\frac{\hat{q} \cdot \nabla T}{T}\right) d A=j T\left(\frac{\partial s}{\partial x}\right)_{j_{e}, j}
$$

The above identity is equivalent to identity (15). In Eq. (19), $\hat{q} \cdot \nabla T=-k_{c}\left(\frac{d T}{d x}\right)^{2}$, where $k_{c}$ is the fluid thermal conductivity. It is remarkable that the integral of Eq. (19) vanishes if and only if the dissipation term vanishes in the case the flow is assumed to be isothermal. On the other hand, for high flow speed, the axial temperature gradient in both, Eq. (18) and Eq. (19), becomes much smaller than the viscous dissipation term. By disregarding the viscous dissipation term in favor of the heat flux term in Eq. (18), Eq. (19) implies that $j_{m}$ becomes constant. Thus Eq. (17) and Eq. (18) enable one to write the following identity,

$$
\left(\frac{\partial j_{e}}{\partial x}\right)_{j_{m}, j}=-\frac{1}{A} \oint_{\Gamma} \hat{q} \cdot \hat{n}_{l} d l=j T\left(\frac{\partial s}{\partial x}\right)_{j_{m}, j}
$$

The above identity is equivalent to identity (16). These equations show that the axial variation of both, the energy density flux and the entropy arise from the heat flux effect at the boundary, as stated in the second part of the Lemma.

Since the pressure is a function of $x$, the following identity also holds,

$$
\left(\frac{\partial j_{e}}{\partial p}\right)_{j}-v\left(\frac{\partial j_{m}}{\partial p}\right)_{j}=j T\left(\frac{\partial s}{\partial p}\right)_{j}
$$

The above equation implies the following identities,

$$
\begin{aligned}
& \left(\frac{\partial j_{m}}{\partial p}\right)_{j_{e}, j}=-\frac{j T}{v} \frac{\partial s}{\partial p}\left(p, j_{e}, j\right) \\
& \left(\frac{\partial j_{e}}{\partial p}\right)_{j_{m}, j}=j T \frac{\partial s}{\partial p}\left(p, j_{m}, j\right)
\end{aligned}
$$


It is remarkable that Eq. (13) suggests that the parameters $j_{e}$ and $j_{m}$ can be regarded as independent thermodynamic variables of the entropy function. Furthermore, the differential form of Eq. (13) enables one to write the following identities,

$$
\begin{aligned}
& \left(\frac{\partial s}{\partial j_{e}}\right)_{j_{m}, j}=\frac{1}{j T} \\
& \left(\frac{\partial s}{\partial j_{m}}\right)_{j_{e}, j}=-\frac{v}{j T}
\end{aligned}
$$

Since $T$ is always positive, Eq. (25) implies that the entropy increases with the increase of the energy density flux, under constant linear momentum density flux. Eq. (26), by its turn shows that the entropy decreases with the increase of the linear momentum density flux, under constant energy density flux.

The proved lemma enables one to prove the following theorem:

Theorem: "In a compressible steady-state fluid flow of a gas in a pipe of constant cross section area, for a given energy flux density, there exist a minimum linear momentum density flux below which a normal shock cannot occur. Conversely, for a given linear momentum density flux, there exist a maximum energy density flux above which a normal shock cannot occur".

Proof: The proof of this theorem follows from the fact that at point $M$ of the state for which $V=c$ the following identity holds

$$
\frac{\partial s}{\partial p}\left(p, j_{e}, j\right)=j^{2} v\left(\frac{\partial v}{\partial s}\right)_{p} \frac{\partial s}{\partial p}\left(p, j_{m}, j\right) /\left[T+j^{2} v\left(\frac{\partial v}{\partial s}\right)_{p}\right]
$$

This identity can be proved with the help of the following identity derived from calculus,

$$
\frac{\partial s}{\partial p}\left(p, j_{m}, j\right)=\frac{\partial s}{\partial p}\left(p, j_{e}, j\right)+\left(\frac{\partial s}{\partial j_{e}}\right)_{p, j}\left(\frac{\partial j_{e}}{\partial p}\right)_{j_{m}, j}
$$

The definition of $j_{e}$ gives,

$$
\left(\frac{\partial j_{e}}{\partial j_{e}}\right)_{p, j}=j\left[\left(\frac{\partial h}{\partial j_{e}}\right)_{p, j}+j^{2} v\left(\frac{\partial v}{\partial j_{e}}\right)_{p, j}\right]=1
$$

where

$$
\left(\frac{\partial h}{\partial j_{e}}\right)_{p, j}=T\left(\frac{\partial s}{\partial j_{e}}\right)_{p, j} \text { and }\left(\frac{\partial v}{\partial j_{e}}\right)_{p, j}=\left(\frac{\partial v}{\partial s}\right)_{p}\left(\frac{\partial s}{\partial j_{e}}\right)_{p, j}
$$

From these three last identities it follows,

$$
\left(\frac{\partial s}{\partial j_{e}}\right)_{p, j}=1 / j\left[T+j^{2} v\left(\frac{\partial v}{\partial s}\right)_{p}\right]
$$

Analogous proof can be used to obtain the following identity

$$
\left(\frac{\partial s}{\partial j_{m}}\right)_{p, j}=1 / j^{3}\left(\frac{\partial v}{\partial s}\right)_{p}
$$


The identity given by Eq. (27) is obtained by replacing in to Eq. (28), the derivative $\left(\frac{\partial j_{e}}{\partial p}\right)_{j_{m}, j}$ by its expression of Eq. (24), and the derivative $\left(\frac{\partial s}{\partial j_{e}}\right)_{p, j}$ by its expression given by Eq. (29). From Eq. (27) one can see that the derivative $\frac{\partial s}{\partial p}\left(p, j_{e}, j\right)$ vanishes if and only if $\frac{\partial s}{\partial p}\left(p, j_{m}, j\right)$ vanishes. Eq. (27) and the results obtained in the previous sections, enable one to conclude that point $M$ is the point for which both, the entropy expressed by $s\left(p, j_{e}, j\right)$ and the entropy expressed by $s\left(p, j_{m}, j\right)$ reach an extremum. It remains to be shown that at point $M, j_{m}$ reaches a minimum while $j_{e}$ reaches a maximum as functions of $p$ for constant $j$.

The second derivative of Eq. (22) leads to the following identity

$$
\left(\frac{\partial^{2} j_{e}}{\partial p^{2}}\right)_{j}-\left(\frac{\partial v}{\partial p}\right)_{j}\left(\frac{\partial j_{m}}{\partial p}\right)_{j}-v\left(\frac{\partial^{2} j_{m}}{\partial p^{2}}\right)_{j}=j\left(\frac{\partial T}{\partial p}\right)_{s}\left(\frac{\partial s}{\partial p}\right)_{j}+j T\left(\frac{\partial^{2} s}{\partial p^{2}}\right)_{j}
$$

For $j_{e}$ constant the above equation is reduced to

$$
\left(\frac{\partial v}{\partial p}\right)_{j_{e}, j}\left(\frac{\partial j_{m}}{\partial p}\right)_{j_{e}, j}+v\left(\frac{\partial^{2} j_{m}}{\partial p^{2}}\right)_{j_{e}, j}=-j\left(\frac{\partial T}{\partial p}\right)_{s}\left(\frac{\partial s}{\partial p}\right)_{j_{e}, j}+j T\left(\frac{\partial^{2} s}{\partial p^{2}}\right)
$$

At point $M$ where $V=c$, Eq. (23) implies that $\left(\frac{\partial j_{m}}{\partial p}\right)_{j_{e}, j}$ vanishes, since $\left(\frac{\partial s}{\partial p}\right)_{j_{e}, j}$ vanishes at the point considered. From the above expression of the second derivative it follows the identity

$$
v\left(\frac{\partial^{2} j_{m}}{\partial p^{2}}\right)=-j T\left(\frac{\partial^{2} s}{\partial p^{2}}\right)_{j_{e}, j}
$$

Since the entropy as a function of $p$ and $j_{e}$ reaches a maximum for $V=c$, its second derivative with respect to $p$ is negative. Therefore the second derivative of the left hand side of the above equation becomes positive. This shows that $M$ is a point of minimum of $j_{m}$ as a function of $p$ and $j_{e}$ for constant $j_{e}$.

For $j_{m}$ regarded as constant, Eq. (24) shows that at point $M,\left(\frac{\partial j_{e}}{\partial p}\right)_{j_{m}, j}$ vanishes, since $\frac{\partial s}{\partial p}\left(p, j_{m}, j\right)$ vanishes at the point considered, while Eq. (31) gives,

$$
\left(\frac{\partial^{2} j_{e}}{\partial p^{2}}\right)_{j_{m}, j}=j\left(\frac{\partial T}{\partial p}\right)_{s}\left(\frac{\partial s}{\partial p}\right)_{j_{m}, j}+j T\left(\frac{\partial s}{\partial p}\right)_{j_{m}, j}
$$

At the point $M$ the first derivative of the entropy vanishes and therefore one has,

$$
\left(\frac{\partial^{2} j_{e}}{\partial p^{2}}\right)_{j_{m}, j}=j T\left(\frac{\partial^{2} s}{\partial p^{2}}\right)_{j_{m}, j}
$$

Since the entropy as a function of $p$ and $j_{m}$ reaches a maximum for $V=c$, its second derivative is negative and therefore the second derivative of the left hand side of the above equation becomes negative. This shows that $M$ is a point of maximum of $j_{e}$ as a function of $p$ and $j_{m}$ for constant $j_{m}$. Figures $2 \mathrm{a}$ and $2 \mathrm{~b}$ show pictures of the tangency point as well as the curves for which two intersecting points are possible, according to the theorem. It should be noted in Fig. $2 \mathrm{~b}$ that $\Delta j_{e}$ is negative. 


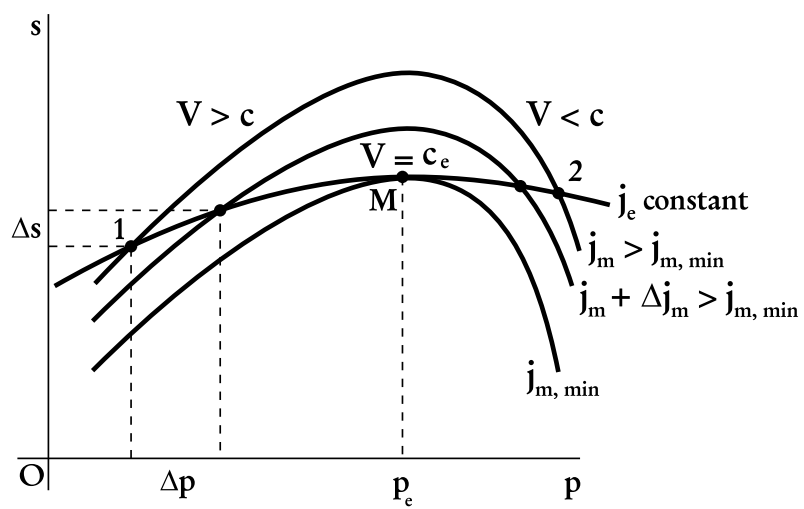

(a) $s=s\left(\mathrm{p}, \mathrm{j}_{\mathrm{e}}, \mathrm{j}\right)$

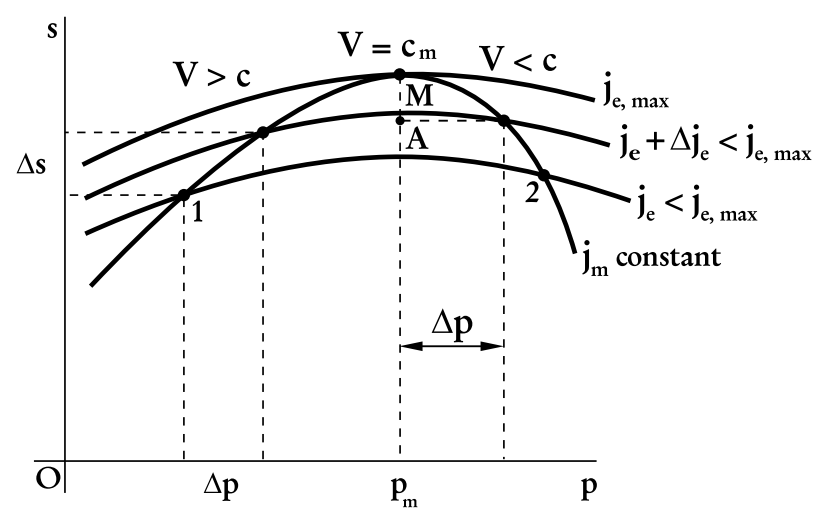

(b) $s=s\left(\mathrm{p}, \mathrm{j}_{\mathrm{m}}, \mathrm{j}\right)$

Figure 2 - (a) Possible states arising from the intersection of Rayleigh curves with a given Fanno curve. (b) Possible states arising from the intersection of Fanno curves with a given Rayleigh curve.

The last step of the proof is to show that point $M$ is a single tangency point of Fanno and Rayleigh curves. By the way, Eq. (6) and Eq. (12) enables one to write the following inequality,

$$
\left.\left(\frac{\partial^{2} s}{\partial p^{2}}\right)_{j_{e}, j}\right|_{V=c}>\left.\left(\frac{\partial^{2} s}{\partial p^{2}}\right)_{j_{m}, j}\right|_{V=c}
$$

On the other hand, in the neighborhood of the tangency point for which $p=p_{e}=p_{m}$, the Taylor expansion of $s\left(p_{e}, j_{e}, j\right)$ gives, $s\left(p_{e} \pm \Delta p, j_{e}, j\right)-s_{\text {max }}\left(p_{e}, j_{e}, j\right)=\left(\frac{\partial^{2} s}{\partial p^{2}}\right)_{j_{e}, j} \frac{(\Delta p)^{2}}{2}$, while the Taylor expansion of $s\left(p_{m}, j_{m}, j\right)$ gives, $s\left(p_{m} \pm \Delta p, j_{m}, j\right)-s_{\text {max }}\left(p_{m}, j_{m}, j\right)=\left(\frac{\partial^{2} s}{\partial p^{2}}\right)_{j_{m}, j} \frac{(\Delta p)^{2}}{2}$. From the above expressions it follows,

$$
s\left(p_{m} \pm \Delta p, j_{e}, j\right)-s\left(p_{m} \pm \Delta p, j_{m}, j\right)=\left[\left(\frac{\partial^{2} s}{\partial p^{2}}\right)_{j_{e}, j}-\left(\frac{\partial^{2} s}{\partial p^{2}}\right)_{j_{m}, j}\right]_{p_{m}} \frac{(\Delta p)^{2}}{2}>0
$$

The above inequality enable on to conclude that the convexity of the curve respective to $s\left(p, j_{m}, j\right)$ (the Rayleigh curve) is greater than the convexity of the curve respective to $s\left(p, j_{e}, j\right)$ (the Fanno curve). From Eq. (33) it can be seen that as $\Delta p$ vanishes, $s\left(p_{m}, j_{m}, j\right)=s\left(p_{m}, j_{e, \max }, j\right)$ and $s\left(p_{m}, j_{e}, j\right)=s\left(p_{m}, j_{m, m i n}, j\right)$, as shown in Fig. 2 .

\section{TEMPERATURE INEQUALITY}

An useful identity to derive an inequality related to the Second Law of thermodynamics can be obtained by casting Eq. (29) with the identity given by,

$$
\left(\frac{\partial T}{\partial j_{e}}\right)_{p, j}=\left(\frac{\partial T}{\partial s}\right)_{p}\left(\frac{\partial s}{\partial j_{e}}\right)_{p, j}
$$


and the thermodynamic identity given by, $\left(\frac{\partial v}{\partial s}\right)_{p}=\frac{T v \alpha}{c_{p}}$. The following identity is found,

$$
\left(\frac{\partial T}{\partial j_{e}}\right)_{p, j}=\frac{1}{j\left(c_{p}+j^{2} v^{2} \alpha\right)}
$$

This identity enables one to conclude that the temperature increases by increasing the energy density flux, under constant pressure. Analogous proof with the help of Eq. (30) leads to the following identity

$$
\left(\frac{\partial T}{\partial j_{m}}\right)_{p, j}=\frac{1}{j^{3} v \alpha}
$$

This identity enables one to conclude that the temperature increases by increasing the linear momentum density flux under constant pressure.

The temperature as a function of $s$ and $p$ gives, $d T=\left(\frac{\partial T}{\partial p}\right)_{s} d p+\left(\frac{\partial T}{\partial s}\right)_{p} d s$, where $\left(\frac{\partial T}{\partial s}\right)_{p}=\frac{T}{c_{p}}$ and $\left(\frac{\partial T}{\partial p}\right)_{s}=\frac{\alpha v T}{c_{p}}$. Integrating the temperature differential between the states of $\left(s_{1}, p_{1}\right)$ and $\left(s_{2}, p_{2}\right)$ it follows,

$$
T_{2}-T_{1}=\int_{p_{1}}^{p_{2}}\left(\frac{\partial T}{\partial p}\right)_{s_{1}} d p+\int_{s_{1}}^{s_{2}}\left(\frac{\partial T}{\partial s}\right)_{p_{2}} d s
$$

The derivative identity respective to $j_{e}$ former to Eq. (34) enables one to write, $\left.\left(\frac{\partial T}{\partial j_{e}}\right)_{p, j} d j_{e}\right|_{p}=\left.\left(\frac{\partial T}{\partial s}\right)_{p} d s\right|_{p}$. Thus, the second term of Eq. (36) can be expressed by $\int_{j_{e}}^{j_{e}+\Delta j_{e}}\left(\frac{\partial T}{\partial j_{e}}\right)_{p_{2}, j} d j_{e}$, for $\Delta j_{e}<0$. Eq. (34) implies that the derivative of $T$ with respect to $j_{e}$ is always positive. From Eq. (36) one can conclude that $T_{2}>T_{1}$ whenever $p_{2}>p_{1}$. One can see from Eq. (34) and Eq. (36) that state (2) can be reached from state (2") by increasing the energy density flux under constant pressure. The chosen integration path is shown in Figure 3.

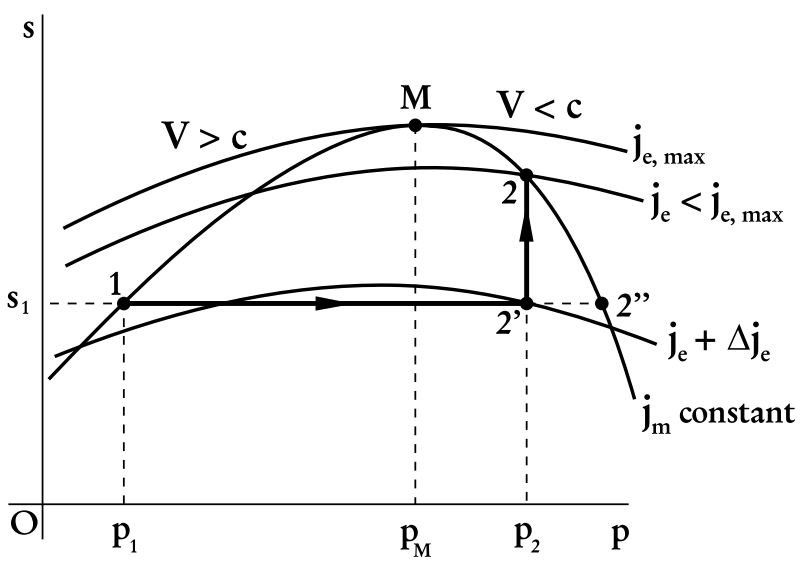

Figure 3 - Integration path respective to Eq. (36). 
Eq. (36) implies that the temperature of state (2") shown in Figure 3, which has the same entropy of state (1), is greater than the temperature of the latter state. In other words, the inequality $T_{2}>T_{1}$ holds whenever $p_{2}>p_{1}$, even if $s_{2}=s_{1}$. Therefore the temperature inequality does not necessarily imply the entropy inequality.

\section{THE MOLLIER DIAGRAM}

The thermodynamic equations expressed in terms of the independent variables $j_{e}$ and $j_{m}$ can be used to derive appropriate equations in order to express the entropy as a function of the enthalpy. Figure 4 illustrates the Fanno and the Rayleigh curves sketched in the well known Mollier diagram.

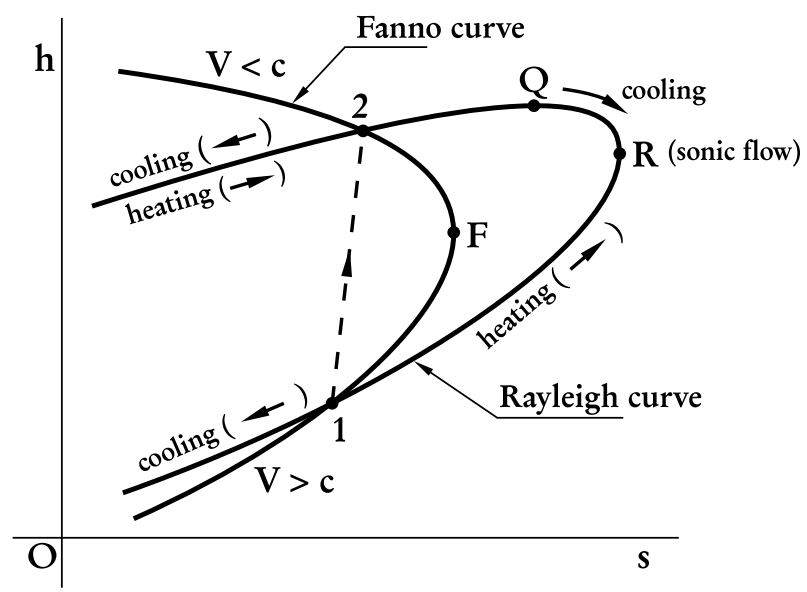

Figure 4 - Fanno and Rayleigh curves in the Mollier diagram.

THE ENTROPY AS A FUNCTION OF $h, j_{e}, \operatorname{AND} j$ - FANNO CURVE

From calculus it follows

$$
\left(\frac{\partial s}{\partial h}\right)_{j_{e}, j}=\left(\frac{\partial s}{\partial p}\right)_{j_{e}, j} /\left(\frac{\partial h}{\partial p}\right)_{j_{e}, j}
$$

while from thermodynamics one has,

$$
\left(\frac{\partial h}{\partial p}\right)_{j_{e}, j}=T\left(\frac{\partial s}{\partial p}\right)_{j_{e}, j}+v
$$

By replacing the derivative of the right hand side of the former equation by its expression given by Eq. (5), it follows,

$$
\left(\frac{\partial h}{\partial p}\right)_{j_{e}, j}=T v\left(\frac{V^{2}}{c^{2}}-1\right) /\left[T+j^{2} v\left(\frac{\partial v}{\partial s}\right)_{p}\right]+v
$$

This derivative can also be expressed as follows

$$
\left(\frac{\partial h}{\partial p}\right)_{j_{e}, j}=v\left[\frac{T V^{2}}{c^{2}}+j^{2} v\left(\frac{\partial v}{\partial s}\right)_{p}\right] /\left[T+j^{2} v\left(\frac{\partial v}{\partial s}\right)_{p}\right]
$$


As remarked earlier, for gases, $\left(\frac{\partial v}{\partial s}\right)_{p}$ is known to be positive. This implies that both, the numerator and the denominator of Eq. (39) are positive. Thus, the inverse derivative $\left(\frac{\partial p}{\partial h}\right)_{j_{e}, j}=1 /\left(\frac{\partial h}{\partial p}\right)_{j_{e}, j}$ becomes also positive. This identity implies that the pressure turns to be a monotonically increasing function of $h$. Thus, one can write, $s=s\left(p, j_{e}, j\right)=s\left(p\left(h, j_{e}, j\right), j_{e}, j\right)=$ $s\left(h, j_{e}, j\right)$, so that this function preserves the shape of the Fanno curve in the $h-s$ Mollier diagram.

As can be seen in Figure 4, point $\mathrm{F}$ is the state for which the entropy reaches a maximum as a function of $h$ for constant $j_{e}$. In fact, Eq. (37) shows that at point $F$, for which $V=c$, the derivative of the entropy as a function of $p$ for constant $j_{e}$ vanishes. Thus, the derivative of the entropy as a function of the enthalpy for constant $j_{e}$ also vanishes.

THE ENTROPY AS A FUNCTION OF $h, j_{m}$, AND $j$ - RAYLEIGH CURVE

From calculus it follows

$$
\left(\frac{\partial s}{\partial h}\right)_{j_{m}, j}=\left(\frac{\partial s}{\partial p}\right)_{j_{m}, j} /\left(\frac{\partial h}{\partial p}\right)_{j_{m}, j}
$$

while from thermodynamics one has $\left(\frac{\partial h}{\partial p}\right)_{j_{m}, j}=T\left(\frac{\partial s}{\partial p}\right)_{j_{m}, j}+v$, where $\left(\frac{\partial s}{\partial p}\right)_{j_{m}, j}$ is given by Eq. (11). The inverse of the derivative given by Eq. (40) is given by the identity,

$$
\left(\frac{\partial h}{\partial s}\right)_{j_{m}, j}=\left(\frac{\partial h}{\partial p}\right)_{j_{m}, j} /\left(\frac{\partial s}{\partial p}\right)_{j_{m}, j}
$$

The above identities and the identity given by Eq. (11) enables one to obtain the following identity,

$$
\left(\frac{\partial h}{\partial s}\right)_{j_{m}, j}=-T\left[\alpha V^{2}-c_{p}\left(1-\frac{V^{2}}{c^{2}}\right)\right] / c_{p}\left(1-\frac{V^{2}}{c^{2}}\right)
$$

For $V$ different from $c$, the denominator of the above equation is non-vanishing. As can be verified, the above derivative vanishes for $V=V_{Q}$, the solution of the algebraic equation given by, $\alpha V^{2}-c_{p}(1-$ $\left.V^{2} / c^{2}\right)=0$. This equation gives,

$$
V_{Q} / c=\sqrt{c_{p} /\left(c_{p}+\alpha c^{2}\right)}
$$

The point $Q$ shown in Fig. 4 represents the state for which the derivative given by Eq. (42) vanishes. For the particular case of a perfect gas, it can be verified that Eq. (43) is reduced to the well known correlation, $V_{Q} / c=1 / \sqrt{k}$. By replacing $c_{p}$ obtained from Eq. (43) into Eq. (42), the following identity is found

$$
\left(\frac{\partial h}{\partial s}\right)_{j_{m}, j}=T\left(\frac{V_{Q}^{2}}{c^{2}}-\frac{V}{c^{2}}\right) /\left(\frac{V_{Q}^{2}}{c^{2}}\right)\left(1-\frac{V^{2}}{c^{2}}\right)
$$

The inverse of the above derivative is expressed as follows

$$
\left(\frac{\partial s}{\partial h}\right)_{j_{m}, j}=\left(1-\frac{V^{2}}{c^{2}}\right)\left(\frac{V_{Q}^{2}}{c^{2}}\right) /\left[T\left(\frac{V_{Q}^{2}}{c^{2}}-\frac{V}{c^{2}}\right)\right]
$$


As shown by Eq. (43), $V_{Q} / c<1$. Therefore, for $V=c$ Eq. (45) leads to $\left(\frac{\partial s}{\partial h}\right)_{j_{m}, j}=0$, which is the necessary condition for point $R$ shown in Fig. 4 to be a point of maximum. Equation (44), by its turn shows that the derivative $\left(\frac{\partial h}{\partial s}\right)_{j_{m}, j}$ vanishes at the point $Q$ shown in the same figure, which is the necessary condition for this point to be a point of maximum. Equation (44) enables one to conclude that for $V<V_{Q}$ and $V<c$, the derivative $\left(\frac{\partial h}{\partial s}\right)_{j_{m}, j}$ is positive, which means the enthalpy increases by increasing the entropy. In other words, the enthalpy increases with the heat income and, therefore, the flow is heated. For any point located between point $Q$ and point $R$ where $V>V_{Q}$ and $V<c$, the derivative $\left(\frac{\partial h}{\partial s}\right)_{j_{m}, j}$ becomes negative, which means the flow is cooled. For any point located at the inner branch of the Rayleigh curve, $V>c$ and $V>V_{Q}$, and therefore the derivative $\left(\frac{\partial h}{\partial s}\right)_{j_{m}, j}$ becomes positive, which means the flow is heated.

\section{THE SECOND LAW INEQUALITY}

The entropy change in the neighborhood of point $M$ of maximum of the entropy, as shown in Fig. 2b, can be expressed in terms of the independent variables $p$ and $j_{e}$, along the curve of $j_{m}$ constant. Since the first derivative $\left(\frac{\partial s}{\partial p}\right)_{j_{e}, j}$ vanishes at point $M$, the Taylor expansion of the entropy up to the second order derivatives evaluated at point $M$, can be expressed as follows

$$
\begin{aligned}
& \left.\Delta s\left(p, j_{e}, j\right)\right|_{j_{m}}= \\
& =\left.\left(\frac{\partial s}{\partial j_{e}}\right)_{p, j} \Delta j_{e}\right|_{j_{m}}+\frac{1}{2}\left(\frac{\partial^{2} s}{\partial p^{2}}\right)_{j_{e}, j}(\Delta p)^{2}+\left.\left(\frac{\partial^{2} s}{\partial p \partial j_{e}}\right)_{j} \Delta_{j_{e}}\right|_{j_{m}} \Delta p+\frac{1}{2}\left(\frac{\partial^{2} s}{\partial j_{e}{ }^{2}}\right)_{p, j}\left(\left.\Delta j_{e}\right|_{j_{m}}\right)^{2}
\end{aligned}
$$

In the above equation $\left.\Delta j_{e}\right|_{j_{m}}$ becomes constrained on the curve of $j_{m}$ constant. The identity given by Eq. (13) leads to, $\left.\Delta j_{e}\right|_{j_{m}}=\left.j T \Delta s\left(p, j_{e}, j\right)\right|_{j_{m}}$. On the other hand, thought $\left(\frac{\partial s}{\partial p}\right)_{j_{m}, j}$ vanishes at point $M$, the Taylor expansion of $s\left(p, j_{m}, j\right)$ in terms of $p$ in the neighborhood of this point is reduced to the following identity,

$$
\left.\Delta s\left(p, j_{e}, j\right)\right|_{j_{m}}=\frac{1}{2}\left(\frac{\partial^{2} s}{\partial p^{2}}\right)_{j_{m}, j}(\Delta p)^{2}
$$

The last two identities lead to, $\left.\Delta j_{e}\right|_{j_{m}}=j T \frac{1}{2}\left(\frac{\partial^{2} s}{\partial p^{2}}\right)_{j_{m}, j}(\Delta p)^{2}$. Since the entropy reaches a maximum at point $M$, its second derivative in this expression is negative and therefore $\left.\Delta j_{e}\right|_{j_{m}}$ becomes negative, as assumed earlier. Moreover $\left.\Delta s\left(p, j_{e}, j\right)\right|_{j_{m}}$ is equal to $\left.\Delta s\left(p, j_{m}, j\right)\right|_{j_{e}}$, for both are equal to the length of the segment $A M$ shown in Fig. 2b. By replacing $\left.\Delta j_{e}\right|_{j_{m}}$ by its last expression and $\left.\Delta s\left(p, j_{e}, j\right)\right|_{j_{m}}$ given by Eq. (47) into Eq. (46), the following algebraic equation of second degree in terms of $\Delta p$ is found,

$$
A(\Delta p)^{2}+B \Delta p+C=0
$$


The coefficients $A, B$, and $C$ evaluated at point $M$ are given as follows,

$$
\begin{aligned}
& A=-\frac{j^{2} T^{2}}{8}\left(\frac{\partial^{2} s}{\partial j_{e}^{2}}\right)_{p, j}\left(\frac{\partial^{2} s}{\partial p^{2}}\right)_{j_{m}, j}^{2} \\
& B=-j T\left(\frac{\partial^{2} s}{\partial j_{e} \partial p}\right)\left(\frac{\partial^{2} s}{\partial p^{2}}\right)_{j_{m}, j} \\
& C=\frac{1}{2}\left[\left(\frac{\partial^{2} s}{\partial p^{2}}\right)_{j_{m}, j}-\left(\frac{\partial^{2} s}{\partial p^{2}}\right)_{j_{e}, j}\right]-\frac{j T}{2}\left(\frac{\partial s}{\partial j_{e}}\right)_{p, j}\left(\frac{\partial^{2} s}{\partial p^{2}}\right)_{j_{m}, j}
\end{aligned}
$$

By taking the derivative of Eq. (29) with respect to $j_{e}$, by reducing the obtained equation with the help of the identity, $\left.\frac{\partial}{\partial j_{e}}\right|_{p, j}=\left.\left(\frac{\partial s}{\partial j_{e}}\right)_{p, j} \frac{\partial}{\partial s}\right|_{p}$, and by replacing the derivative $\left(\frac{\partial s}{\partial j_{e}}\right)_{p, j}$ by its expression of Eq. (29), the following identity is found

$$
\left(\frac{\partial^{2} s}{\partial j_{e}^{2}}\right)_{p, j}=-\left[\left(\frac{\partial T}{\partial s}\right)_{p}+j^{2}\left(\frac{\partial v}{\partial s}\right)_{p}^{2}+j^{2} v\left(\frac{\partial^{2} v}{\partial s^{2}}\right)_{p}\right] / j^{2}\left[T+j^{2} v\left(\frac{\partial v}{\partial s}\right)_{p}^{3}\right.
$$

The second derivative in the numerator of the above equation can be replaced by the identities,

$$
\frac{\partial}{\partial s}\left(\frac{\partial v}{\partial s}\right)_{p}=\frac{\partial}{\partial T}\left(\frac{\partial v}{\partial s}\right)_{p}\left(\frac{\partial T}{\partial s}\right)_{p}=\frac{\partial}{\partial T}\left(\frac{\alpha T v}{c_{p}}\right)_{p} \frac{T}{c_{p}}
$$

The term of the last equality can be expanded in the form

$$
\left(\frac{\partial^{2} v}{\partial s^{2}}\right)_{p}=\frac{T v}{c_{p}^{2}}\left\{\alpha+\alpha^{2} T+T\left[\left(\frac{\partial \alpha}{\partial T}\right)_{p}-\frac{\alpha}{c_{p}}\left(\frac{\partial c_{p}}{\partial T}\right)_{p}\right]\right\}
$$

Eq. (51) and Eq. (52) enable one to express Eq. (48) as follows

$$
A=-\frac{\frac{T^{3}}{8 c_{p}}\left(\frac{\partial^{2} s}{\partial p^{2}}\right)_{j_{m}, j}^{2}\left\{1+\frac{j^{2} v^{2}}{c_{p}}\left[\alpha+T\left(\frac{\partial \alpha}{\partial T}\right)_{p}\right]+\frac{j^{2} v^{2} T \alpha}{c_{p}}\left[2 \alpha-\frac{1}{c_{p}}\left(\frac{\partial c_{p}}{\partial T}\right)_{p}\right]\right\}}{\left[T+j^{2} v\left(\frac{\partial v}{\partial s}\right)_{p}\right]^{3}}
$$

In the above equation, $j^{2} v^{2}=V^{2}=c^{2}$ while from thermodynamics it is known that, $c^{2}=\frac{v c_{p}}{c_{v} k_{T}}$. Thus the above equation can also be expressed as follows,

$$
A=\frac{\frac{1}{8}\left(\frac{\partial^{2} v}{\partial p^{2}}\right)_{s}^{2} \frac{c_{p}}{T^{2} \alpha^{2} v^{2}}\left\{1+\frac{v}{c_{v} k_{T}}\left[\alpha+T\left(\frac{\partial \alpha}{\partial T}\right)_{p}\right]+\frac{\alpha T v}{c_{v} k_{T}}\left[2 \alpha-\frac{1}{c_{p}}\left(\frac{\partial c_{p}}{\partial T}\right)_{p}\right]\right\}}{\left(1+\frac{\alpha v}{c_{v} k_{T}}\right)^{3}}
$$

For the particular case of an ideal gas, for which $\alpha=\frac{1}{T}$, the expression into the first bracket of the numerator of the above equation vanishes, while the remaining terms are reduced to the following,

$$
1+\frac{\alpha T v}{c_{v} k_{T}}\left[2 \alpha-\frac{1}{c_{p}}\left(\frac{\partial c_{p}}{\partial T}\right)_{p}\right]
$$


By replacing $\alpha, c$, and $k_{T}$, this expression can be reduced to,

$$
\frac{1}{c_{v o}}\left[c_{p o}+R-\frac{R T}{c_{p o}} \frac{d c_{p o}}{d T}\right]
$$

As the author has verified, this expression is positive for all ideal gases currently reported in thermodynamics textbooks.

By taking the derivative of Eq. (4) with respect to parameter $j_{e}$, at point $M$ the following identity holds,

$$
\frac{\partial^{2} s}{\partial j_{e} \partial p}=-j v \frac{\partial^{2} v}{\partial p \partial s} /\left[T+j^{2} v\left(\frac{\partial v}{\partial s}\right)_{p}\right]^{2}
$$

where, $\frac{\partial^{2} v}{\partial p \partial s}=\frac{\partial}{\partial s}\left(-k_{s} v\right)_{p}=-\left[v\left(\frac{\partial k_{s}}{\partial s}\right)_{p}+k_{s}\left(\frac{\partial v}{\partial s}\right)_{p}\right],\left(\frac{\partial k_{s}}{\partial s}\right)_{p}=\left(\frac{\partial k_{s}}{\partial T}\right)_{p}\left(\frac{\partial T}{\partial s}\right)_{p}$, $\left(\frac{\partial v}{\partial s}\right)_{p}=\frac{\alpha T v}{c_{p}}>0$, and $\left(\frac{\partial T}{\partial s}\right)_{p}=\frac{T}{c_{p}}>0$. From these identities it follows

$$
\frac{\partial^{2} v}{\partial p \partial s}=-\frac{T v}{c_{p}}\left[k_{s} \alpha+\left(\frac{\partial k_{s}}{\partial T}\right)_{p}\right]
$$

Equations (49), (55), and (56) enable one to write constant $B$ as follows

$$
B=\frac{c_{p}}{c_{v} k_{T} \alpha T}\left(\frac{\partial^{2} v}{\partial p^{2}}\right)_{s}\left[k_{s} \alpha+\left(\frac{\partial k_{s}}{\partial T}\right)_{p}\right] /\left(1+\frac{\alpha v}{c_{v} k_{T}}\right)^{2}
$$

For the particular case of a perfect gas, $k_{s}=1 / k p$, where $k$ is the Poisson coefficient. Thus, $\left(\frac{\partial k_{s}}{\partial T}\right)_{p}=0$. By considering that the second derivative in the numerator of Eq. (57) is positive, for this particular case considered, the constant $B$ becomes positive. One can see from Eq. (57) that the constant $B$ is positive if and only if the inequality, $k_{s} \alpha+\left(\frac{\partial k_{s}}{\partial T}\right)_{p}>0$ holds.

By replacing the derivatives in Eq. (50) by their expressions given by Eq. (6) and Eq. (12), the following equation is found,

$$
C=-\frac{T}{2}\left(\frac{\partial^{2} v}{\partial p^{2}}\right)_{s} /\left(\frac{\partial v}{\partial s}\right)_{p}\left[T+j^{2} v\left(\frac{\partial v}{\partial s}\right)_{p}\right]
$$

By replacing the derivative $\left(\frac{\partial v}{\partial s}\right)_{p}=\frac{\alpha T v}{c_{p}}$ in the above equation it follows

$$
C=-\frac{1}{2} \frac{c_{p}}{\alpha T v}\left(\frac{\partial^{2} v}{\partial p^{2}}\right)_{s} /\left(1+\frac{\alpha v}{c_{v} k_{T}}\right)
$$

Since the second derivative of the above equation is assumed to be positive, the coefficient $C$ becomes unconditionally negative. The roots of the formerly obtained algebraic equation in terms of are given by, $\Delta p^{-}=-\left(B+\sqrt{B^{2}-4 A C}\right) /(2 A)$ and $\Delta p^{+}=-\left(B-\sqrt{B^{2}-4 A C}\right) /(2 A)$. Let us assume that $k_{s} \alpha+\left(\frac{\partial k_{s}}{\partial T}\right)_{p}>0$ holds, in which case $B$ becomes positive. By assuming the constant $A$ to be negative, one 
has, $\sqrt{B^{2}-4 A C}<B$, since $C<0$. Thus, $\Delta p^{-}$becomes positive. Since by definition $\Delta p^{-}=p^{-}-p_{M}$, $p^{-}$becomes grater than $p_{M}$, which cannot be the case. Therefore, $A$ must be positive. Thus Eq. (54) implies the inequality given below

$$
1+\frac{v}{c_{v} k_{T}}\left[\alpha+T\left(\frac{\partial \alpha}{\partial T}\right)_{p}\right]+\frac{\alpha T v}{c_{v} k_{T}}\left[2 \alpha-\frac{1}{c_{p}}\left(\frac{\partial c_{p}}{\partial T}\right)_{p}\right]>0
$$

Since $C$ is negative one has $\sqrt{B^{2}-4 A C}>B$, which implies $\Delta p^{+}>0$ and $\Delta p^{-}<0$ as expected. Moreover, $\Delta p^{+}+\Delta p^{-}=-B / A$. It follows from this inequality that $\left|\Delta p^{-}\right|>\Delta p^{+}$. On the other hand, Eq. (47) leads to, $s^{-}-s_{M}=\left.\Delta s^{-}\right|_{j_{m}}=\frac{1}{2}\left(\frac{\partial^{2} s}{\partial p^{2}}\right)_{j_{m}, j}\left(\Delta p^{-}\right)^{2}$, and $s^{+}-s_{M}=\left.\Delta s^{+}\right|_{j_{m}}=\frac{1}{2}\left(\frac{\partial^{2} s}{\partial p^{2}}\right)_{j_{m}, j}\left(\Delta p^{+}\right)^{2}$. These equations enable one to write the following,

$$
s^{+}-s^{-}=s^{+}-s_{M}-\left(s^{-}-s_{M}\right)=\frac{1}{2}\left(\frac{\partial^{2} s}{\partial p^{2}}\right)_{j_{m}, j}\left[\left(\Delta p^{+}\right)^{2}-\left(\Delta p^{-}\right)^{2}\right]
$$

Since at point $M$ the second derivative of the entropy is negative, the above equation implies, $s^{+}>s^{-}$. This inequality shows that the entropy increases during the transition of a weak shock, from the supersonic state of $s^{-}$to the subsonic state of $s^{+}$. Conversely, by assuming the Second Law inequality to hold for a weak shock, as is currently assumed, Eq. (61) clearly implies the inequality, $\left(\Delta p^{-}\right)^{2}>\left(\Delta p^{+}\right)^{2}$. As can be shown, this inequality leads to, $B \sqrt{B^{2}-4 A C}>0$ and therefore, $B>0$. From Eq. (57) follows the inequality, $k_{s} \alpha+\left(\frac{\partial k_{s}}{\partial T}\right)_{p}>0$.

From the identity given by Eq. (25), for the states of $s^{-}$and $s^{+}$on the curve of constant $j_{m}$ one has, $\left(\frac{\partial s}{\partial j_{e}}\right)_{j_{m}, j}^{-}=\frac{1}{j T^{-}}$and $\left(\frac{\partial s}{\partial j_{e}}\right)_{j_{m}, j}^{+}=\frac{1}{j T^{+}}$. It should be taken into account that along the curve of constant $j_{m}$, for both states, $s\left(p, j_{m}, j\right)=s\left(p, j_{e}, j\right)$ and therefore in each branch of the curve given by $s\left(p, j_{m}, j\right)$, the later equality leads to an implicit function of $j_{m}, j_{e}$, and $p$, for constant $j$. As Fig. 5 shows, for a given value of $j_{e}$, the curve of constant $j_{m}$ crosses the curve of constant $j_{e}$ in two distinct points corresponding to two states, one of which defined by $p^{-}$, which is a supersonic state and the other one defined by $p^{+}$, which is a subsonic state.

The former derivatives of the entropy enable one to write the following inequality

$$
\left(\frac{\partial s}{\partial j_{e}}\right)_{j_{m}, j}^{-}=\frac{1}{j T^{-}}>\frac{1}{j T^{+}}=\left(\frac{\partial s}{\partial j_{e}}\right)_{j_{m}, j}^{+}
$$

for Eq. (36) gives $T^{+}-T^{-}>0$ for $p^{+}>p^{-}$and $s^{+} \geq s^{-}$.

By multiplying both sides of inequality (62) by an arbitrarily small $\left(-\left.\Delta j_{e}\right|_{j_{m}}\right)>0$, the following inequality is obtained,

$$
s_{M}-s^{-} \approx\left(\frac{\partial s}{\partial j_{e}}\right)_{j_{m}, j}^{-}\left(-\left.\Delta j_{e}\right|_{j_{m}}\right)>\left(\frac{\partial s}{\partial j_{e}}\right)_{j_{m}, j}^{+}\left(-\Delta j-\left.e\right|_{j_{m}}\right) \approx s_{M}-s^{+}
$$




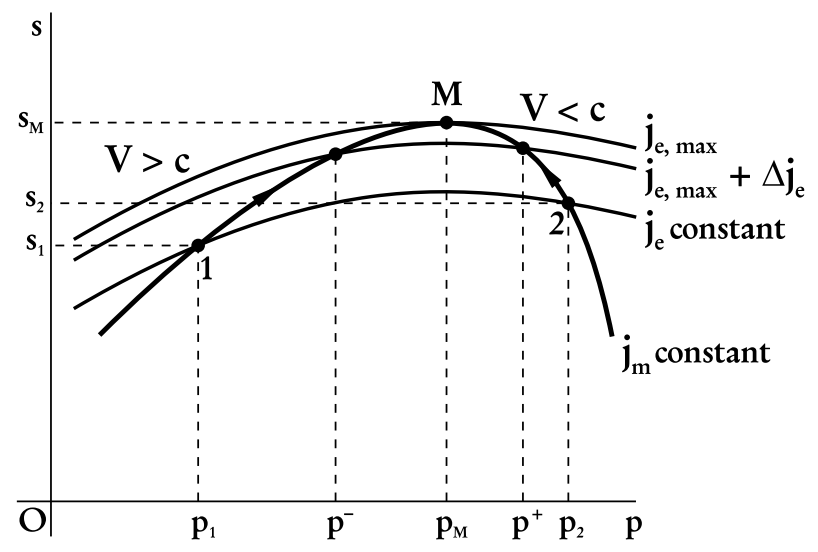

Figure 5 - Integration path for inequality (63).

The above inequality implies $s^{+}>s^{-}$. On the other hand, by integrating the left hand side of inequality (62) from state (1) to state of $s^{-}$and the right hand side of the same inequality from the state of to state (2) with respect to the variable $j_{e}$ along the path shown in Fig. 1 it follows

$$
\begin{gathered}
s^{-}-s_{1}=\int_{j_{e}}^{j_{e, \max }+\Delta j_{e}}\left(\frac{\partial s}{\partial j_{e}}\right)_{j_{m}, j}^{-} d j_{e}=\int_{j_{e}}^{j_{e, \max }+\Delta j_{e}} \frac{1}{j T^{-}} d j_{e}> \\
>\int_{j_{e}}^{j_{e, \max }+\Delta j_{e}} \frac{1}{j T^{+}} d j_{e}=\int_{j_{e}}^{j_{e, \max }+\Delta j_{e}}\left(\frac{\partial s}{\partial j_{e}}\right)_{j_{m}, j}^{+} d j_{e}=s^{*}-s_{2}
\end{gathered}
$$

where $\Delta j_{e}=\left.\Delta j_{e}\right|_{j_{m}}<0$. This inequality gives, $s_{2}>s_{1}+\left(s^{+}-s^{-}\right)>s_{1}$ and therefore, $s_{2}>s_{1}$. The above inequality shows that as $\Delta j_{e}$ vanishes, as shown in Fig. 5 both, $s^{-}$and $s^{+}$become equal to $s_{M}$, and therefore the above inequality is reduced to the following

$$
s_{M}-s_{1}=\int_{j_{e}}^{j_{e, \max }}\left(\frac{\partial s}{\partial j_{e}}\right)_{j_{m}, j}^{-} d j_{e}>\int_{j_{e}}^{j_{e, \max }}\left(\frac{\partial s}{\partial j_{e}}\right)_{j_{m}, j}^{+} d j_{e}=s_{M}-s_{2}
$$

The above inequality also implies $s_{2}>s_{1}$. These results enable one to conclude that the entropy discontinuity arising from a weak shock implies an entropy discontinuity for a strong shock.

\section{EVALUATION OF THE TANGENCY POINT}

At the tangency point the following equations must be imposed

$$
\begin{aligned}
\left(\frac{\partial s}{\partial p}\right)\left(p, j_{m}, j\right) & =0 \\
\left(\frac{\partial s}{\partial p}\right)\left(p, j_{e}, j\right) & =0 \\
s\left(p, j_{m}, j\right) & =s\left(p, j_{e}, j\right)
\end{aligned}
$$

Equation (64) can be written as

$$
\left(\frac{\partial s}{\partial p}\right)_{v}+\left(\frac{\partial s}{\partial v}\right)_{p}\left(\frac{\partial v_{m}}{\partial p}\right)_{j_{m}, j}=0
$$


From the definition of $j_{m}$ it follows

$$
v_{m}=\left(j_{m} / j-p\right) / j^{2}
$$

Equation (65) can be written as

$$
\left(\frac{\partial s}{\partial p}\right)_{v}+\left(\frac{\partial s}{\partial v}\right)_{p}\left(\frac{\partial v_{e}}{\partial p}\right)_{j_{e}, j}=0
$$

From the definition of $j_{e}$ it follows

$$
\varphi=h\left(p, v_{e}\right)+j^{2} v_{e} / 2-j_{e} / j=0
$$

The derivative $\left(\frac{\partial v_{e}}{\partial p}\right)_{j_{e}, j}$ in Eq. (69) is determined by the identities arising from calculus,

$$
\begin{gathered}
\left(\frac{\partial v_{e}}{\partial p}\right)_{j_{e}, j, \varphi}=-\left(\frac{\partial \varphi}{\partial p}\right)_{v, j_{e}, j} /\left(\frac{\partial \varphi}{\partial v}\right)_{p, j_{e}, j},\left(\frac{\partial \varphi}{\partial p}\right)_{v, j_{e}, j}=\left(\frac{\partial h}{\partial p}\right)_{v}, \text { and } \\
\left(\frac{\partial \varphi}{\partial v}\right)_{p, j_{e}, j}=\left(\frac{\partial h}{\partial v}\right)_{p}+j^{2} v .
\end{gathered}
$$

By replacing these derivatives into Eq. (69) it follows

$$
\left[\left(\frac{\partial h}{\partial v}\right)_{p}+j^{2} v\right]\left(\frac{\partial s}{\partial p}\right)_{v}-\left(\frac{\partial h}{\partial p}\right)_{v}\left(\frac{\partial s}{\partial v}\right)_{p}=0
$$

On the other hand, from thermodynamics one has,

$$
\begin{aligned}
& \left(\frac{\partial s}{\partial v}\right)_{p}=\frac{c_{p}}{\alpha v T},\left(\frac{\partial s}{\partial p}\right)_{v}=\frac{c_{v} k_{T}}{\alpha T}, d h=T d s+v d p \\
& \left(\frac{\partial h}{\partial p}\right)_{v}=T\left(\frac{\partial s}{\partial p}\right)_{v}+v=\frac{c_{v} k_{T}}{\alpha}+v,\left(\frac{\partial h}{\partial v}\right)_{p}=T\left(\frac{\partial s}{\partial v}\right)_{p}=\frac{c_{p}}{\alpha v}
\end{aligned}
$$

Furthermore, Eq. (68) gives, $\left(\frac{\partial v_{m}}{\partial p}\right)_{j_{m}}=-\frac{1}{j^{2}}$. By casting these identities with Eq. (71) the following identity is obtained

$$
j^{2} v_{m} c_{v} k_{T}-c_{p}=0
$$

This identity can also be obtained from Eq. (67). Therefore, at the tangency point the following equations must be satisfied

$$
\begin{aligned}
p+j^{2} v-j_{m} / j & =0 \\
h(p, v)+j^{2} v^{2} / 2-j_{e} / j & =0 \\
j^{2} v c_{v} k_{T}-c_{p} & =0
\end{aligned}
$$

These equations can be simultaneously solved in term of $p$, $v$, and $j_{e}$ for a specified value of $j_{m}$ or in terms of $p, v$, and $j_{m}$ for a given value of $j_{e}$. The identity given by Eq. (75), as can be verified, implies $V=c$ 
for, $j^{2} v^{2}=V^{2}$ and $c^{2}=c_{p} v /\left(c_{v} k_{T}\right)$. The details of the analytical solutions obtained for the particular case of a perfect gas are given in the Appendix.

Another interesting identity to show that $j_{e}$ and $j_{m}$ play the role of independent variables can be derived from the Jacobian of these parameters respective to $p$ and $v$, as defined by,

$$
J\left(\frac{j_{e}, j_{m}}{p, v}\right)=\left(\frac{\partial j_{e}}{\partial p}\right)_{v, j}\left(\frac{\partial j_{m}}{\partial v}\right)_{p, j}-\left(\frac{\partial j_{e}}{\partial v}\right)_{p, j}\left(\frac{\partial j_{m}}{\partial p}\right)_{v, j}
$$

where $\left(\frac{\partial j_{e}}{\partial p}\right)_{v, j}=j\left(\frac{\partial h}{\partial p}\right)_{v},\left(\frac{\partial j_{e}}{\partial v}\right)_{p, j}=j\left[\left(\frac{\partial h}{\partial v}\right)_{p}+j^{2} v\right],\left(\frac{\partial j_{m}}{\partial p}\right)_{v, j}=j,\left(\frac{\partial j_{m}}{\partial v}\right)_{p, j}=j^{3}$, $\left(\frac{\partial h}{\partial v}\right)_{p}=\frac{c_{p}}{\alpha v}$, and $\left(\frac{\partial h}{\partial p}\right)_{v}=\frac{c_{v} k_{T}}{\alpha}+v$. By replacing the above derivatives in the Jacobian given by Eq. (76) it follows,

$$
J\left(\frac{j_{e}, j_{m}}{p, v}\right)=\frac{j\left(j^{2} v c_{v} k_{T}-c_{p}\right)}{\alpha v}
$$

It is remarkable that this Jacobian vanishes at the point $M$, for Eq. (75) holds at this point. This means that at point $M, j_{m}$ and $j_{e}$ become conjugated, as shown by the theorem proved here.

\section{ANALYTICAL EXAMPLE FOR A PERFECT GAS}

In order to illustrate the analysis presented in this paper, the particular case of a perfect gas will be presented. For a perfect gas, the enthalpy can be expressed by $h=k p v /(k-1)$, where $k=c_{p o} / c_{v o}$ is assumed equal to 1,4 . The physical variables can be replaced by the dimensionless variables, $\alpha=p / p_{o}, \mu=j^{2} v / p_{o}$, $\zeta_{m}=\left(s_{m}-s_{o m}\right) / R, \zeta_{e}=\left(s_{e}-s_{o m}\right) / R=\left(s_{e}-s_{o e}\right) / R+\left(s_{o e}-s_{o m}\right) / R$, where $s_{e}=s\left(p, j_{e}, j\right)$, $s_{m}=s\left(p, j_{m}, j\right)$, and $s_{o e}$ and $s_{o m}$ are the values of the entropy at an arbitrary chosen state given by $p_{o}$ and $v_{o}$. The dimensionless enthalpy is defined by $\chi=h j^{2} / p_{o}$. Let us define also, $\beta_{m}=j_{m} /\left(j p_{o}\right)$ and $\beta_{e}=j j_{e} / p_{o}^{2}$. The corresponding dimensionless equations respective to equations presented in the previous section are given in the Appendix. Fig. 6a shows the Fanno and Rayleigh curves in a dimensionless entropy - pressure diagram, for the case for which $\beta_{m}$ is specified.

In Fig. 7, $\beta_{m}=1,05 \beta_{m, \min }$ and $\beta_{e}=0,95 \beta_{e, \max }$ were chosen in order to fit the curves in a reduced diagram size. In the present case it is found, $\chi_{A}=1,225, \chi_{R}=1,164, \alpha_{Q}=0,6388, \chi_{Q}=1,389$, $\zeta_{Q}=2,846$, and $\frac{V_{Q}}{c}=\frac{1}{\sqrt{k}}$.

The curves are plotted for $\beta_{m}=1,2$, for which $\beta_{e, \max }=1,47$ and $\alpha_{e}=0,5$ are found. For the sake of comparison, the curve of $\beta_{e}=0,8 \beta_{e, \max }$ is also plotted. Fig. $2 \mathrm{~b}$ shows the Fanno and Rayleigh curves for the case for which $\beta_{e}$ is specified. The curves are plotted for $\beta_{e}=1,47$, for which $\beta_{m, \min }=1,2$ and $\alpha_{m}=0,5$ are found. For the sake of comparison, the curve of $\beta_{m}=1,1 \beta_{m, \text { min }}$ is also plotted. These particular numerical cases are chosen in order to show the evaluation of the unknown parameters are correctly computed. Fig. 7a and $7 \mathrm{~b}$ show the Fanno and Rayleigh curves plotted in the dimensionless Mollier diagram, corresponding to Fig. $6 \mathrm{a}$ and $6 \mathrm{~b}$. 

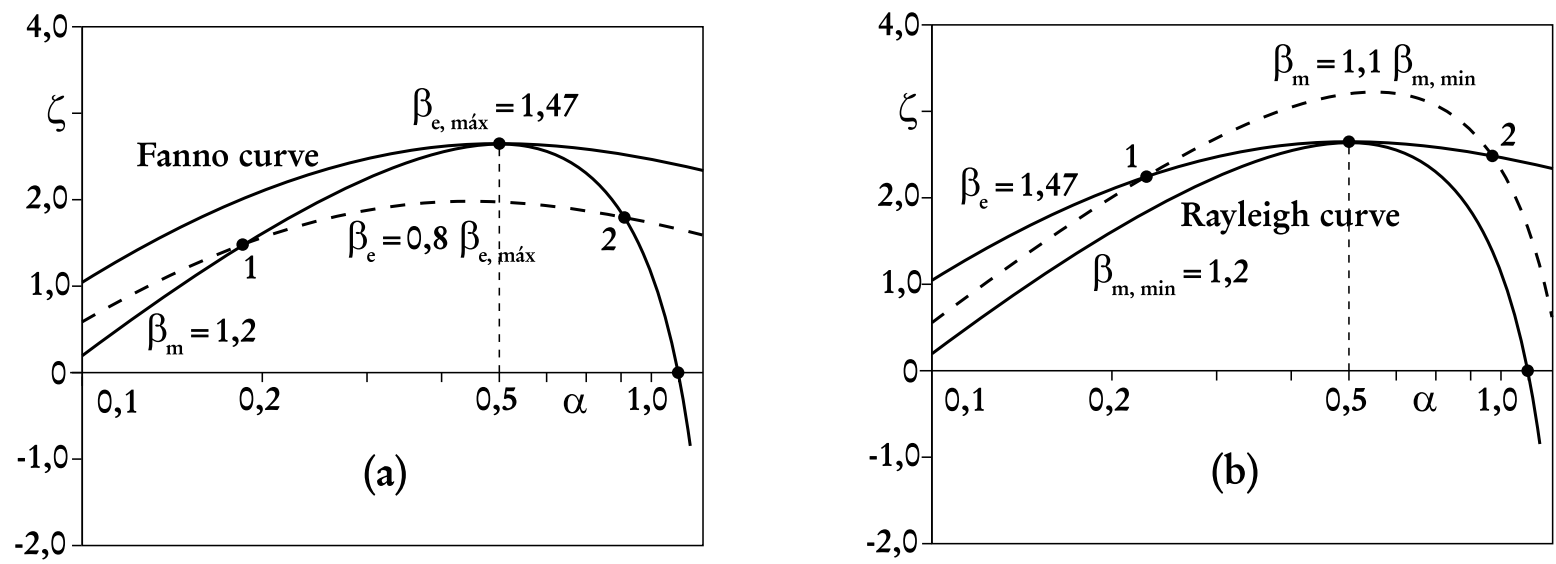

Figure 6 - (a) Limiting Rayleigh curve for a given Fanno curve. (b) Limiting Fanno curve for a given Rayleigh curve.
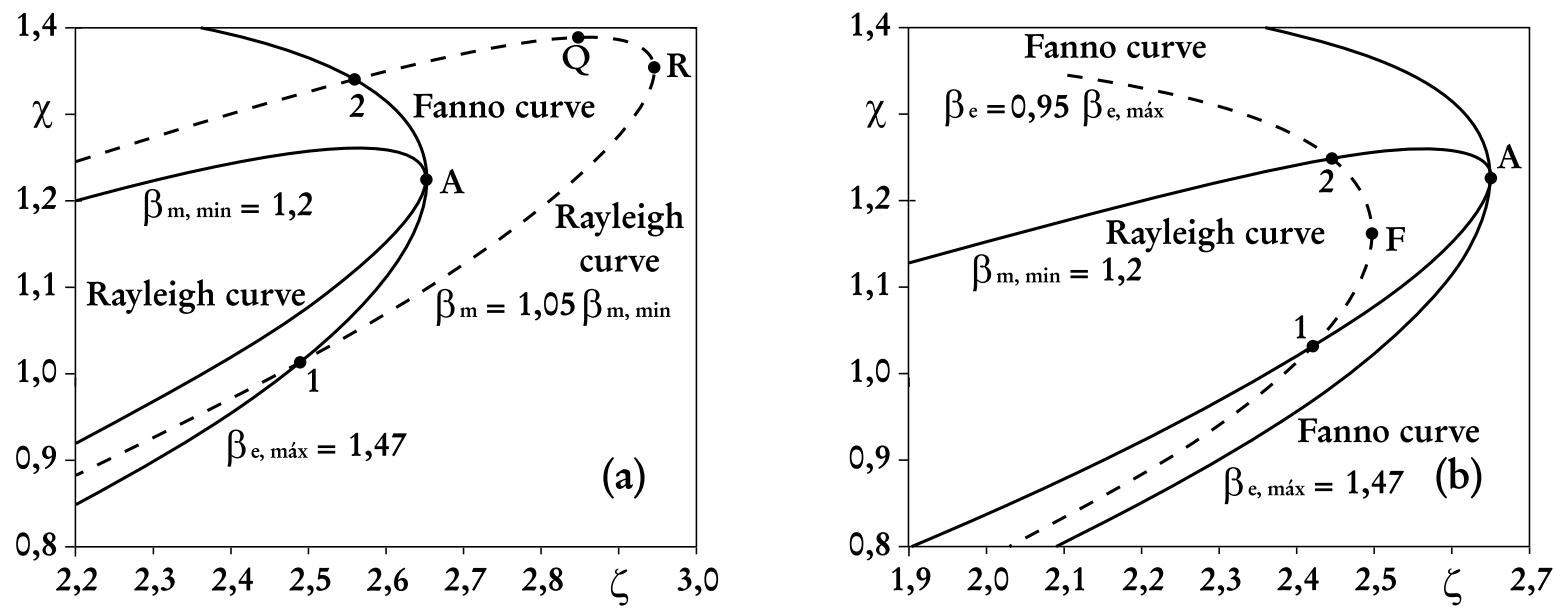

Figure 7 - (a) Limiting Rayleigh curve for a given Fanno curve in the Mollier diagram. (b) Limiting Fanno curve for a given Rayleigh curve in the Mollier diagram.

\section{CONCLUSIONS}

In the present paper, it is shown that the Fanno flow and Rayleigh flow can be thermodynamically related in order to set up the condition under which a normal shock can occur in compressible flow of a gas in a pipe. Thermodynamic identities are proved in order to show that there is a single point where the Fanno and the Rayleigh curves become tangent to each other. It is also shown that the contact and tangency point of the Rayleigh and Fanno curves is the single thermodynamic state for which Fanno flow and Rayleigh flow become dependent of each other, trough an existing relationship among the linear momentum density flux, the energy density flux, and the pressure. It is also shown that the linear momentum density flux and the energy density flux can play a role as independent thermodynamic properties of the entropy function. It is shown also that a Second Law inequality can be obtained by expressing the entropy both as a function of the linear momentum density flux and as a function of the energy density flux, respectively to Rayleigh and 
Fanno flows. The theoretical results are graphically illustrated for the particular case of a perfect gas in both, the $s-p$ and the Mollier diagram.

\section{ACKNOWLEDGMENTS}

The author is indebted to Ministry of Science and Technology for the researcher fellowship of Conselho Nacional de Desenvolvimento Científico e Tecnológico (CNPq). The author is grateful to his doctor student Allan R. Starke for his help with the computation of the equations. The author is also greatful to the support provided by project ANEEL/PETROBRAS No. PD-0553-0023/2012.

\section{REFERENCES}

ANDERSON JD. 2003. Modern compressible flow: with historical perspective, $3^{\text {rd }}$ ed., McGraw-Hill. BAR-MEIR G. 2013. Fundamentals of Compressible Flow Mechanics, version 0.4.9.8. Free e-book. COLLE S. 2015. Lectures on Classical Thermodynamics: Volume V - Compressible Fluid Flow (in portuguese), Edition of the Departament of Mechanical Engineering - Federal University of Santa Catarina, Florianópolis, Brazil, ISBN 978-85-916577-5-9.

LANDAU LD AND LIFSHITZ EM. 1959. Mechanics of Fluids, 1st ed., London: Pergamon Press. RAYLEIGH JWS. 1899-1920. Scientific Papers. 6 Vols, Cambridge, England.

SHAPIRO AH. 1953. The Dynamics and Thermodynamics of Compressible Fluid Flow. Vol. 1, Ronald Press.

\section{APPENDIX}

The enthalpy of a perfect gas can be expressed as $h=k p v /(k-1)$. By this definition Eq. (74) becomes

$$
\left(\frac{k}{k-1}\right) p v+\frac{j^{2} v^{2}}{2}-\frac{j_{e}}{j}=0
$$

From Eq. (75) follows $j^{2} v k_{T}-k=0$ where $k_{T}=1 / p$ and therefore

$$
p=j^{2} v / k
$$

By replacing $v$ from its expression given by Eq. (73) in the above equation it follows

$$
p_{m}=j_{m} /[j(k+1)]
$$

By combining this equation with Eq. (79) one has

$$
v_{m}=k j_{m} /\left[j^{3}(k+1)\right]
$$

From the equation of the sound speed given by, $c^{2}=k p v$, Eq. (79), and Eq. (80) it follows

$$
c_{m}=k j_{m} /\left[j^{2}(k+1)\right]
$$

From Eq. (78) follows

$$
v_{e}=\frac{1}{j^{2}}\left[\sqrt{\left(\frac{k}{k-1}\right)^{2} p^{2}+2 j j_{e}}-\left(\frac{k}{k-1}\right) p\right]
$$


By replacing $v_{e}$ from the above equation in Eq. (79) it follows

$$
p_{e} \frac{\sqrt{2}}{k} \sqrt{\frac{k-1}{k+1}} \sqrt{j j_{e}}
$$

By eliminating $p_{e}\left(=p_{m}\right)$ from Eq. (80) and Eq. (84) the following function relating $j_{m}$ and $j_{e}$ is found

$$
j_{m}=\frac{\sqrt{2}}{k} j \sqrt{k^{2}-1} \sqrt{j j_{e}}
$$

From Eq. (79) and Eq. (84) follows

$$
v_{e}=\frac{\sqrt{2}}{j^{2}} \sqrt{\frac{k-1}{k+1}} \sqrt{j j_{e}}
$$

From the sound speed equation given by $c^{2}=k p v$, Eq. (84), and Eq. (86) it follows

$$
c_{e}=\sqrt{2} \sqrt{\frac{k-1}{k+1}} \sqrt{\frac{j_{e}}{j}}
$$

By dividing both sides of Eq. (73) by $p_{o}$ one has, $j_{m} /\left(j p_{o}\right)=p / p_{o}+j^{2} v / p_{o}$. By defining the dimensionless variables $\beta_{m}=j_{m} /\left(j p_{o}\right), \alpha=p / p_{o}$, and $j^{2} v / p_{o}$, Eq. (68) is reduced to, $\beta_{m}=\alpha+\mu$, from which comes

$$
\mu_{m}\left(\alpha, \beta_{m}\right)=\beta_{m}-\alpha
$$

where $\mu_{m}=j^{2} v_{m} / p_{o}, \mu_{o m}=j^{2} v_{o m} / p_{o}$, and $\mu_{o m}=\beta_{m}-1$, and therefore $\beta_{m}>1$ and $v_{m}>1$. Eq. (88) implies $\alpha<\beta_{m}$. Equation (78) can be reduced to the dimensionless form, $\beta_{e}=\left(\frac{k}{k-1}\right) \alpha \mu+\frac{\mu^{2}}{2}$ where $\beta_{e}=j j_{e} / p_{o}^{2}$ is dimensionless. Solving the former equation in terms of $\mu$ one has,

$$
\mu_{e}\left(\alpha, \beta_{e}\right)=\sqrt{\left(\frac{k}{k-1}\right) \alpha^{2}+2 \beta_{e}}-\left(\frac{k}{k-1}\right) \alpha
$$

where $\mu_{e}=j^{2} v_{e} / p_{o}$, so that the above equation implies, $\beta_{e}>0$. By making $v_{e}=v_{m}$, Eq. (81) and Eq. (86) give, $\frac{k j_{m}}{j^{3}(k+1)}=\frac{\sqrt{2}}{j^{2}} \sqrt{\frac{k-1}{k+1}} \sqrt{j j_{e}}$, from which it follows, $\frac{j_{m}}{j}=\frac{\sqrt{2}}{k} \sqrt{k^{2}-1} \sqrt{j j_{e}}$. By dividing both sides of this equation by $p_{o}$, the following dimensionless function relating $\beta_{e}$ and $\beta_{m}$ is found

$$
\beta_{m}=\frac{\sqrt{2}}{k} \sqrt{k^{2}-1} \sqrt{\beta_{e}}
$$

At the point of maximum of both, $s\left(p, j_{m}, j\right)$ and $s\left(p, j_{e}, j\right)$, Eq. (80) and Eq. (84) give respectively

$$
\begin{aligned}
\alpha_{m} & =\frac{\beta_{m}}{(k+1)} \\
\alpha_{e} & =\frac{\sqrt{2}}{k} \sqrt{\frac{k-1}{k+1}} \sqrt{\beta_{e}}
\end{aligned}
$$

The entropy function can be expressed as follows,

$$
s_{m}\left(p, j_{m}, j\right)=s_{o m}+c_{p o} \ln \left(T / T_{o}\right)-R \ln \left(p / p_{o}\right)
$$


By replacing $T$ and $T_{o}$ in terms of the pressure and volume it follows

$$
s_{m}\left(p, j_{m}, j\right)=s_{o m}+c_{p o} \ln \left[p v_{m} /\left(p_{o} v_{o m}\right)\right]-R \ln \left(p / p_{o}\right)
$$

where $v_{m}$ is expressed by Eq. (68) and $v_{o m}$ is expressed by the same equation in the form

$$
v_{\text {om }}=\left(j_{m} / j-p_{o}\right) / j^{2}
$$

In the same way,

$$
s_{e}\left(p, j_{e}, j\right)=s_{o e}+c_{p o} \ln \left[p v_{e} /\left(p_{o} v_{o e}\right)\right]-R \ln \left(p / p_{o}\right)
$$

where $v_{e}$ is expressed by Eq. (83) and $v_{o m}$ is expressed by the same equation in the form

$$
v_{o e}=\frac{1}{j^{2}}\left[\sqrt{\left(\frac{k}{k-1}\right)^{2} p_{o}^{2}+2 j j_{e}}-\left(\frac{k}{k-1}\right) p_{o}\right]
$$

The dimensionless entropies are defined by $\zeta_{m}=\left(s_{m}-s_{o m}\right) / R$, and

$$
\zeta_{e}=\left(s_{m}-s_{o m}\right) / R=\left(s_{e}-s_{o e}\right) / R+\left(s_{o e}-s_{o m}\right) / R
$$

Thus, Eq. (93) and Eq. (95) can be expressed by their respective dimensionless forms as follows

$$
\begin{array}{r}
\zeta_{m}\left(\alpha, \beta_{m}\right)=\left(\frac{k}{k-1}\right) \ln \left[\frac{\alpha \mu_{m}\left(\alpha, \beta_{m}\right)}{\mu_{m}\left(1, \beta_{m}\right)}\right]-\ln \alpha \\
\zeta_{e}\left(\alpha, \beta_{m}\right)=\Delta \zeta_{e}+\left(\frac{k}{k-1}\right) \ln \left[\frac{\alpha \mu_{e}\left(\alpha, \beta_{e}\right)}{\mu_{e}\left(1, \beta_{e}\right)}\right]-\ln \alpha
\end{array}
$$

where $\Delta \zeta_{e}=\left(s_{o e}-s_{o m}\right) / R, \mu_{m}\left(\alpha, \beta_{m}\right)$ is expressed by Eq. (88) and $\mu_{e}\left(\alpha, \beta_{e}\right)$ is expressed by Eq. (89).

Two cases are analyzed as follows.

\section{Case (a) - The parameter $\boldsymbol{\beta}_{\boldsymbol{m}}>1$ is specified}

From Eq. (90) one has $\beta_{e, \max }=\beta_{m}{ }^{2} k^{2} /\left[2\left(k^{2}-1\right)\right]$, and from Eq. (91) one has $\alpha_{m}=\beta_{m} /(k+1)$. The contact condition for the entropy curves given by $s_{m}$ and $s_{e}$, for the present case is assured by the equality $\zeta_{m}\left(\alpha_{m}, \beta_{m}\right)=\zeta_{e}\left(\alpha_{m}, \beta_{e, \max }\right)$. From this equation, Eq. (97), and Eq. (98) it follows

$$
\Delta \zeta_{e}\left(\alpha_{m}\right)=\left(\frac{k}{k-1}\right) \ln \left[\frac{\mu_{m}\left(\alpha_{m}, \beta_{m}\right) \mu_{e}\left(1, \beta_{e, \max }\right)}{\mu_{m}\left(1, \beta_{m}\right) \mu_{e}\left(\alpha_{m}, \beta_{e, \max }\right)}\right]
$$

The contact of the curve of constant $\beta_{e}$ with the curve of constant $\beta_{m}$ is assured by expressing Eq. (98) as follows

$$
\zeta_{e}\left(\alpha, \beta_{e}\right)=\Delta \zeta_{e}\left(\alpha_{m}\right)+\left(\frac{k}{k-1}\right) \ln \left[\frac{\alpha \mu_{e}\left(\alpha, \beta_{e}\right)}{\mu_{e}\left(1, \beta_{e, \max }\right)}\right]-\ln \alpha
$$

while $\zeta_{m}\left(\alpha, \beta_{m}\right)$ is expressed by Eq. (97).

\section{Case (b) - The parameter $\beta_{e}$ is specified}


From Eq. (90) one has $\beta_{m, \text { min }}=\frac{\sqrt{2}}{k} \sqrt{k^{2}-1} \sqrt{\beta_{e}}$, and from Eq. (92) one has $\alpha_{e}=\frac{\sqrt{2}}{k} \sqrt{\frac{k-1}{k+1}} \sqrt{\beta_{e}}$. The contact condition for the entropy curves is assured by the equality $\zeta_{m}\left(\alpha_{e}, \beta_{m, \min }\right)=\zeta_{e}\left(\alpha_{e}, \beta_{e}\right)$. From this equation, Eq. (97), and Eq. (98) it follows

$$
\Delta \zeta_{e}\left(\alpha_{e}\right)=\left(\frac{k}{k-1}\right) \ln \left[\frac{\mu_{m}\left(\alpha_{e}, \beta_{m, \min }\right) \mu_{e}\left(1, \beta_{e}\right)}{\mu_{m}\left(1, \beta_{m, \min }\right) \mu_{e}\left(\alpha_{e}, \beta_{e}\right)}\right]
$$

The contact of the curve of constant $\beta_{e}$ with the curve of constant $\beta_{m}$ is assured by expressing Eq. (97) as follows

$$
\zeta_{m}\left(\alpha, \beta_{m}\right)=\left(\frac{k}{k-1}\right) \ln \left[\frac{\alpha \mu_{m}\left(\alpha, \beta_{m}\right)}{\mu_{m}\left(1, \beta_{m, m i n}\right)}\right]-\ln \alpha
$$

while $\zeta_{e}\left(\alpha, \beta_{e}\right)$ is expressed by Eq. (98).

The point $Q$ located on the Rayleigh curve as shown in Fig. 4 is determined by Eq. (86) given in the same reference. For a perfect gas, $V_{Q} / c_{s}=1 / \sqrt{k}$. By replacing $V_{Q}=j v_{Q}$ in the preceding equation one has, $j v_{Q} / c_{s}=1 / \sqrt{k}$. By replacing the sound speed $c_{s}$ from its expression of Eq. (82) in the last equation one has,

$$
j^{3} v_{Q}(k+1) / j_{m}=\sqrt{k}
$$

In terms of the dimensionless variables the above equation is reduced to the following equation,

$$
\left(\beta_{m}-\alpha_{Q}\right) / \beta_{m}=\sqrt{k} /(k+1)
$$

This equation gives $\alpha_{Q}=\beta_{m}(1+k-\sqrt{k}) /(k+1)$.

The dimensionless enthalpy is defined as

$$
\chi=h j^{2} / p_{o}^{2}
$$

From the expressions of $\mu$ and $\alpha$ one has,

$$
\chi=k \mu \alpha /(k-1)
$$

By the definition $\mu$ and $\alpha$, at the point $Q$, this equation leads to the following equation

$$
\chi_{Q}=k \alpha_{Q}\left(\beta_{m}-\alpha_{Q}\right) /(k-1)
$$

